\title{
Desafios enfrentados pelos idosos nas unidades de saúde
}

\author{
Challenges faced by the elderly in health care facilities \\ Retos de las personas mayores en los centros sanitarios
}

Leonardo Felipe de Sampaio Belotto ${ }^{1 *}$, Patricia Dupont ${ }^{1}$, Siegrid Kurzawa Zwiener dos Santos².

\begin{abstract}
RESUMO
Objetivo: Expor as dificuldades relacionadas à comunicação enfrentadas pela terceira idade no SUS. Revisão bibliográfica: O Brasil está tendo o mesmo destino dos países desenvolvidos, ou seja, está aumentando a expectativa de vida, causando o envelhecimento populacional, sendo assim, a saúde pública deve se adaptar à essa nova realidade. Alguns autores relatam que os idosos superestimam o atendimento na Atenção Primaria à Saúde (APS), isso faz com que se tornem dependentes do sistema. Além disso, outros estudos apontam que é necessário analisar o paciente idoso de maneira sistêmica, já que, por muitas vezes seu acompanhante pode omitir informações, caso o paciente esteja sendo violentado e/ou seja dependente. Considerações finais: Diante de tais motivações encontradas, faz-se necessário um trabalho socioeducativo com os profissionais e com a população com o intuito de demonstrar como observar a linguagem corporal, além de expor as opções de melhoria ao cuidado integral ao paciente idoso, melhorando assim, a relação médico-paciente idoso.
\end{abstract}

Palavras-chave: Saúde do idoso, Assistência integral à saúde, Violência ao idoso, Idoso dependente.

\begin{abstract}
Objective: To expose the difficulties related to communication faced by the elderly in the SUS. Bibliographic review: Brazil is facing the same fate as developed countries, i.e., it is increasing life expectancy, causing population aging, so public health must adapt to this new reality. Some authors report that the elderly overestimate the care in Primary Health Care (PHC), which makes them dependent on the system. In addition, other studies point out that it is necessary to analyze the elderly patient in a systemic way, since, many times, the companion may omit information, if the patient is being abused and/or is dependent. Final considerations: Given these motivations, a socio-educational work with professionals and the population is necessary in order to demonstrate how to observe body language, as well as to expose the options for improving the integral care of the elderly patient, thus improving the physician-patient relationship.
\end{abstract}

Keywords: Health of the elderly, Comprehensive health care, Elderly violence, Dependent elderly.

\section{RESUMEN}

Objetivo: Exponer las dificultades relacionadas con la comunicación que enfrentan los ancianos en el SUS. Revisión bibliográfica: Brasil está teniendo el mismo destino que los países desarrollados, es decir, está aumentando la esperanza de vida, provocando el envejecimiento de la población, por lo que la salud pública debe adaptarse a esta nueva realidad. Algunos autores señalan que los ancianos sobrevaloran la atención en la Atención Primaria de Salud (APS), lo que les hace depender del sistema. Además, otros estudios señalan que es necesario analizar al paciente anciano de forma sistémica, ya que, en muchas ocasiones su acompañante puede omitir información, si el paciente está siendo maltratado y/o es dependiente. Consideraciones finales: Debido a estos motivos encontrados, se hace necesario un trabajo socioeducativo con los profesionales y con la población con la intención de demostrar cómo observar el lenguaje corporal, además de mostrar las opciones de mejora del cuidado integral al paciente ídem, mejorando así, la relación médico-paciente.

Palabras clave: Salud del anciano, Atención sanitaria integral, Violencia en el anciano, Ancianos dependientes.

${ }_{1}^{1}$ Universidade do Contestado (UnC), Mafra - SC. *E-mail: leonardo31052001@hotmail.com

2 Universidade Federal do Paraná (UFPR), Curitiba - PR.

SUBMETIDO EM: $8 / 2021$

ACEITO EM: 8/2021

PUBLICADO EM: 8/2021 


\section{INTRODUÇÃO}

O Brasil está atualmente vivendo o Bônus demográfico, ou seja, o país está envelhecendo. Isto está ocorrendo graças aos avanços relacionados à expectativa de vida. Entretanto, o Sistema Público de Saúde não se encontra preparado para tal evolução. Por conta deste processo de envelhecimento, o sistema de saúde deve passar por adequações da oferta de serviços e da qualidade de recursos humanos. De modo que o sistema seja adaptado para atender a todas as necessidades e garantir o acesso da pessoa idosa (RAMOS LV, et al., 2019; LUBENOW JAM, et al., 2019; ALMEIDA APSC, et al., 2020).

Concomitantemente a isso, é notório que em nosso país os principais usuários do sistema de saúde são os idosos, por conseguinte, a interdependência entre ambos é muito notória no nosso país em comparação aos demais países do mundo. Tal fato se deve ao aumento de doenças crônicas e a confiança entre o paciente e o profissional (LUBENOW JAM, et al., 2019; COELHO LP, et al., 2019).

Outrossim, o profissional da saúde deve receber preparo, uma vez que, o idoso necessita muitas vezes cuidados domiciliares. Desse modo, expondo a vulnerabilidade do paciente. Sendo assim, a fim de melhorar a qualidade de vida do mesmo, a equipe multiprofissional deve propor um plano farmacológico, que possibilite que o paciente usufrua corretamente deste (POZZOLI SML, et al., 2017; MORAES EM, et al., 2019).

Em vista disso, a Atenção Primária a Saúde (APS) possui certas diretrizes, como por exemplo a longitunalidade, integralidade e acessibilidade, ou seja, seu propósito é amenizar as filas em locais de grandes complexidades, diminuir as sobrecargas do profissional, além de oferecer um atendimento digno e integral a todos usuários. Em consequência a isso, todos os funcionários atuantes na APS devem se submeter a um plano para que todas as diretrizes, anteriormente citadas, se desenvolvam de maneira integral e correta (SCHENKE M, et al., 2019).

Diante de tal premissa, o presente artigo teve como principal objetivo demonstrar as maiores dificuldades da comunicação do médico com o paciente idoso no sistema de saúde do Brasil. De modo em que demonstre a todos os leitores uma reflexão a respeito da temática abordada em conjunto com a síntese de estudos.

\section{REVISÃO BIBLIOGRÁFICA}

Temos o conhecimento de que o Brasil vive o bônus demográfico, não sendo surpresa que o país está seguindo os passos de países desenvolvidos: o envelhecimento populacional. Por tal motivo, o acesso da pessoa idosa à APS deve ser permeado a Organização do cuidado, por conseguinte, prevenir agravos crônicos dos brasileiros. Desse modo, permitir que esses mantenham a independência e a autonomia com eficácia e responsabilidade (RAMOS LV, et al., 2019; MORAES EM, et al., 2019).

Concomitante a isso, devido a particularidade de cada idoso, como será abordado pelo presente trabalho, se faz necessário que tanto os profissionais como os cuidadores estejam preparados para a realização do presente auxílio. De modo em que, não sobrecarregue ambos trabalhadores e diminua as demandas para as Unidade de Alta Complexidade (CARDOSO SMM, et al., 2020).

Ademais, a incorporação da Rede de Atenção a Saúde (RAS) é um processo complexo, já que o intuito é analisar os idosos de maneira sistêmica. Sendo assim, averiguar toda a rede do idoso se faz necessário para manter a saúde mais estável possível, dando melhores condições de saúde (MORAES EM, et al., 2019).

Por outro lado, o envelhecimento do país e o atendimento focado apenas em uma determinada patologia do paciente idoso estão presentes em nosso cotidiano e tais motivações interferem na resolubilidade da rede de saúde e negligência, principalmente, o paciente idoso dependente. Em suma, estas pessoas em situações de vulnerabilidade que, em sua grande maioria, precisam de cuidados paliativos com medidas de conforto, porém não recebem tais cuidados em casa devido a falta de empatia e preparação da equipe multidisciplinar (SILVA RM, et al., 2021; MARQUES FP, et al., 2018).

Porém, sabe-se que o processo de envelhecimento pode ser tanto natural quanto biológico, por vários fatores que ocorrem no corpo das pessoas e durante as fases das nossas vidas. Mas, se compreende que apenas os presentes fatores não contribuem suficientemente para definir o ciclo da velhice e sua história. 
Acredita-se que os fatores sociais, ambientais, culturais e psicossociais das pessoas ao longo dos tempos, devem ser considerados para a compreensão integral do tal processo. $O$ aumento do percentual da população idosa, está diretamente relacionado com a diminuição da taxa de natalidade e mortalidade infantil, melhores condições de saneamento básico, acesso aos serviços hospitalares e de saúde, como também o avanço nos tratamentos de doenças infectocontagiosas (SOUSA EA, et al., 2019).

Entretanto, a integralidade do cuidado em saúde deve envolver a multidisciplinariedade, adaptando-se a cada fase da vida, promovendo a saúde e a independência do indivíduo, a qual será atingida com o acesso total aos recursos existentes na Unidade Básica de Saúde. Além disso, vivenciar os momentos limitados de outrem sob seus cuidados, mesclando diversos aspectos emocionais e técnicos, esses antecedentes fazem da relação trabalho em saúde na prática da atenção básica a base do pensamento e da discussão na atualidade (SILVA RM, et al., 2021; MARQUES FP, et al., 2018).

\section{A violência vivenciada por idosos}

A violência à pessoa idosa possui como definição qualquer ação que altere o estado de saúde da vítima, seja ela física ou mental, realizada de forma única ou repetida, ou ainda, omissão das necidades da pessoa idosa, ocorrida diante de uma relação em que se espere uma confiança, que acarrete um certo problema a uma pessoa idosa. Entretanto, com base em muitos estudos, sabe-se que o indivíduo idoso fica mais vulnerável à violência na medida em que se tormam mais dependentes de terceiros, seja para cuidados físicos ou mental. Além de que, percebe-se que a relação entre a dependência e o grau de vulnerabilidade são diretamente proporcionais (CASTRO VC, et al., 2017; OLIVEIRA KSM, et al., 2017).

Por outro lado, o declínio das habilidades funcionais e cognitivas do idoso, histórias de relações de violências, dependência e sobrecarga econômica, estresse e transtôrno psicopatológico do cuidador, podem acarretar em violência contra o idoso. Portanto, é difícil estimar as ocorrências e as consequências da violência contra o idoso, pois as fontes de dados são escassas e, as vezes, pouco confiáveis, visto que o evento é encoberto por familiares e pela sociedade. Os profissionais só podem identificar as vítimas de violência, quando os mesmos comentam coisas que podem indicar algo ou por meio das informações fornecidas pelos agentes de saúde que a identificam no local onde o idoso mora ou até mesmo na própria vizinhança da vítima (BARROS RLM, et al., 2019; OLIVEIRA KSM, et al., 2017).

Ademais, os idosos maltratados sentem a agressão desde um abuso verbal, até a agressão física, enquanto o agressor nem sempre percebe que esse tipo de comportamento é violento, como por exemplo, não trocar a fralda urinada ou simplesmente não dar um copo de água ao idoso (OLIVEIRA KSM, et al., 2017).

Em suma, a violência ao idoso é um problema relacionado com a saúde pública no Brasil, pois representa um imenso impacto nos sistemas de saúde, previdenciário e de segurança pública, ou seja, a violência a presente população causa uma gravidade multisetorial, levando a uma péssima qualidade de vida das pessoas (CASTRO VC, et al., 2017).

\section{Perspectiva do idoso à UBS}

As Unidades Básicas de Saúde (UBS), que incluem as Unidades de Saúde da Família (USF), têm como papel fundamental na resobilidade da imensa maioria das patologias, cerca de $80 \%$. Ainda, se observa que no Brasil, aproximadamente $75 \%$ (setenta e cinco por cento) da população utiliza exclusivamente do SUS. Desse modo, o tal sistema se constitui na porta de entrada entre saúde e usuário. Contudo, a contrução de modelos de atenção para pacientes idosos portadores de doenças crônicas tem se mostrado um equívoco, uma vez que com tempo, a fisiologia corporal se torma mais dependente (PLACIDELI N e NEMES MIB, 2020).

Ademais, evidências cientificas demontram que com o passar da idade é relativamente comum os sintomas de depressão, ansiedade e angústia devido ao pensamento de morte eminente e abandono dos familiares e amigos. Sendo assim, se faz necessário um preparo por parte dos profissionais da Atenção primária para que seja possível abordar esse idoso. Tendo como resultado a redução dos agravos causados pela patologia em questão. Posteriomente, elevando a qualidade de vida e a autonomia do paciente idoso, e junto a isso, melhorando a qualidade da rede de relações sociais do mesmo (RAMOS FP, et al., 2019). 
Outrossim, percebe-se que nas UBS, muitos idosos idealizam seus atendimentos, realizando a transferência na figura médica. Além de que, a medida em que a população vai envelhecendo, precisa de mais atenção e otimismo, já que é através de tais que o envelhecimento será saudável, por conseguinte, a divinização do médico não será necessária. Por isso, os profissionais da área da saúde têm como obrigação analisar toda a rede do idoso, uma vez que, se o idoso imagina e cria expectativa sobre o atendimento, ou até mesmo relate algo que dê um entendimento de negligência, o profissional poderá conduzir a seu atendimento de uma maneira mais adequada (LUBENOW JAM, et al., 2019; TAVARES RE, et al., 2017).

Ademais, os determinantes da utilização de serviços de saúde estão relacionados a fatores contextuais, como por exemplo os tipos de sistemas de saúde e sua organização e a fatores individuais. Além de que, temos o conhecimento que o idoso tem influência de suas crenças e seu psicológico, sendo assim, o uso da UBS se torna um meio de lidar com os problemas psicossociais. Destarte, os fatores individuais compreendem características predisponentes, (como idade e sexo), características facilitadoras (como escolaridade e renda) e necessidades de saúde. Desse modo, tais características podem afetar positivamente ou negativamente no processo saúde e doença, uma vez que, algumas morbidades apresentam tais fatores como classificação de risco (SILVA AMM, et al., 2017; TAVARES RE, et al., 2017).

\section{Desafios enfrentado pelos idosos frente à APS}

O rápido crescimento da população idosa vai além do Brasil, ou seja, é uma realidade mundial, sendo assim, configura-se um desafio para a saúde pública da contemporaneidade. Concomitantemente, observase que alguns idosos são dependentes para o acompanhamento médico, portanto, é necessário avaliar os aspectos psicossociais do paciente, uma vez que é através dos gestos que o profissional poderá avaliar a veracidade das informações passadas pelo acompanhante (THUM C, et al., 2019; BANDEIRA EMFS, et al., 2014).

Durante todo o processo de cuidado de um paciente precisa envolver profissionais de diferentes áreas da saúde e preparo qualificado da mesma, ou seja, todos necessitam compreender como funciona cada etapa do ciclo de vida, para atingir um objetivo em comum, o alívio total do paciente, para que gere um sentimento de indepencia por parte do paciente (SILVA RM, et al., 2021).

Ademais, estudos apontam que a veracidade nas informações passadas pelo profissional durante a consulta médico-paciente idoso é um instrumento terapêutico de extrema importância. Desse modo, pesquisas sugerem que tais informações diminuem a sensação de isolamento, dando assim um sentimento de cooperação e mutualismo (VIANNA LG, et al., 2009; LUBENOW JAM, et al., 2019).

Pesquisas afirmam que a grande maioria dos pacientes idosos relatam que a APS apresenta inúmeras burocracias, sendo os mais comuns: metas de segurança ao paciente inatingidas, gestão ineficiente, falta de empatia por parte da equipe, filas de espera longas, falhas estruturais e organizacionais e falta de segurança pública. Portanto, ao analisar tais dificuldades, tanto na contrução da APS, como do manejo das consultas, o paciente idoso cria em sua mente certas hipóteses diagnósticas, especulando a gravidade da sua possível doença e, por conseguinte, acaba criando barreiras durante o seu uso da UBS (SILVA RM, et al., 2021; VIANNA LG, et al., 2009).

\section{Problemas do sistema de saúde}

Existemas diversas formas de atendimento no Brasil para a pessoa idosa, como por exemplo,a internação hospitalar, o atendimento ambulatorial especializado, o hospital-dia, Atenção primária em Saúde (APS), Estratégia da Saúde da Família (ESF), Rede de Atenção em Saúde (RAS), Unidades de Pronto Atendimento (UPAs), Unidade Básica de Saúde (UBS) e a assistência domiciliar. Os ambientes anteriormente citados, buscam aliviar todo e qualquer prejuízo a saúde da pessoa idosa, sendo que os mais presentes nesta faixa etária são: insuficiência cardíaca, doenças pulmonares obstrutivas crônicas (DPOCs) e doenças cerebrovasculares (BENITO LAO e AQUINO EB, 2016; RODRIGUES MM, et al., 2019).

Entretanto, estudos qualitativos realizados no Brasil apontam que a média de espera para conseguir uma internação em locais de grande complexidade são idênticos quando comparados os convênios, SUS ou 
particular, concentrando entre 0 a 3 dias de espera. No entando, as mesmas pesquisas comprovam que 0 paciente idoso que necessita de internação pelos Sistema Único de Saúde obtivaram mais tempo na fila de espera quando comparados aos pacientes não idosos, sendo em média mais de 31 dias de espera. Outrossim, a análise integral de estudos, traz que a população idosa, a qual foi encaminhada pelas UBS, possui mais chances de ter morbidade crônica, além de ter mais chances de ir para as Unidades de Terapia Intensiva (UTI) por falta de médico mais qualificado em observar sistematicamente as patologias (ALMEIDA APSC, et al, 2020; RÊGO AS, et al., 2017).

Com base em evidências científicas, se entende que um grande problema das Unidades Básicas de Saúde e da APS é adaptar seus centros para atender a demanda de idosos que precisam de atendimento. Desse modo, as literaturas trazem que tal problema é advindo da dificuldade dos idosos em contratar planos de saúde devido as altas taxas de mensalidades, das burocracias e, por conseguinte acabam dependendo exclusivamente dos serviços do SUS (SOUSA EA, et al., 2019).

Além do que foi supracitado, estudos apontam que os idosos trazem consigo um gasto elevado por internações, uma vez que são mais vulneráveis fisiologicamente, socialmente e psicologicamente, ou seja, devido as consequências do envelhecimento, o paciente idoso acaba se tornando mais dependente de internações para que possa ter mais atenção (RÊGO AS, et al., 2017).

Destarte, tanto no sistema público quanto no privado é preciso uma integração de todos os níveis de atenção, uma vez que se isso ocorrer haverá um atendimento continuado e holístico, por conseguinte, o sistema apresentará integralidade da atenção para o usuário. Sendo assim, usufruir apenas de tratamentos medicamentosos traz uma enorme dependência, além de consequências bioquímicas do idoso; todavia, sabese que atualmente a terapia medicamentosa em certos casos é a única solução, ou seja, a articulação do cuidado necessita de maiores avaliações (ALMEIDA APSC, et al., 2020; ANDRADE, et al., 2021).

\section{Assistência farmacêutica no cuidado ao idoso}

Devido ao envelhecimento do povo brasileiro há um aumento da demanda dos investimentos na reformulação de novas formas de cuidados prolongados, sejam estes em casa ou no atendimento domiciliar realizados pelos serviços públicos de saúde. No entanto, observa-se que ocorrem certos conflitos entre o idoso e seus cuidadores, como por exemplo desavenças familiares, cobrança social, falta de diálogo entre o cuidor e o idoso, falta de informações sobre o problema físico e psicológico do paciente e desconhecimento do manuseio e das técnicas inerentes ao cuidado, falha na colaboração do paciente e falta de recursos, sejam estes estruturais ou não (MARQUES FP, et al., 2018; POZZOLI SML, et al., 2017).

De acordo com a Classificação Brasileira de Ocupações do Ministério do Trabalho e Emprego, uma nova profissão que foi conhecida é a de cuidador de idosos. São considerados indivíduos Cuidadores de Pessoas Idosas e Dependentes e Cuidador de Idosos Institucional, as pessoas que realmente cuidam do idoso, mantém o bem-estar, alimentação, higiene pessoal, saúde e diversão da pessoa em que estão cuidando, deixando-a se sentir confortável na sua residência o máximo possível (CARDOSO SMM, et al.,2020).

Ademais, com o advento da Rede de Atenção a Saúde (RAS), a integralidade no atendimento ocorreu de maneira mais rápida e ágil; uma vez que com a ferramenta da visita domiciliar houve uma aproximação entre o profissional e o paciente, dado assim aos familiares uma sensação de conforto ao cuidar de um idoso dependente. Deste modo, o atendimento domiciliar como modelo assistencial possibilita que a promoção e prevenção em saúde sejam exercidos em espaço familiar e agradavél, com significado afetivo para o paciente dependente e que, garante um certo grau da autonomia do idoso. Porém, verifica-se que é no momento do atendimento domiciliar que são identificadas todas as falhas no cuidado do paciente idoso, e também se observa a necessidades de cuidados paliativos, bem como o funcionamento integral das relações familiares importantes no processo de saúde-doença (MARQUES FP, et al., 2018; POZZOLI SML, et al., 2017).

Muitos idosos continuam trabalhando e fazendo suas tarefas domésticas, auxiliando na produtividade da sociedade atual. No entando, a partir do final do século XX, fez-se necessário no cenário nacional a figura do cuidador de idosos, pois uma parte significativa dessa população idosa, possui uma ou múltiplas condições físicas dependentes ou alguma doença orgânica debilitante. Com o passar dos anos, houve um aumento de 
doenças crônicas na sociedade atual, evidenciando a importância dos cuidadores de idosos. Porém, percebese que a terceira idade está mais vulnerável a violência, negligência e abandono, ficando assim, dependentes dos cuidadores CARDOSO SMM, et al., 2020).

Indentifica-se que os prestadores de serviço na atenção domiciliar se deparam, com certa frequência, idosos em situação de fragilidade, que, muitas vezes, esses pacientes não possuem consciência de que estão sendo vítimas de terceiros. Outrossim, ao entrar na realidade de um usuário limitado, ser recebido na casa e realizar ações de saúde, as quais o profissional que está lá para ofertar de todas as formas possíveis a melhor estratégia de cuidado, que por conseguinte resultará em uma aproximação humana, sendo esta positiva para a relação médico-paciente (POZZOLI SML, et al., 2017; MARQUES FP, et al., 2018).

Todavia, percebe-se assim, o quanto o papel das esquipes de ESFs são importante no treinamento e supervisão desses cuidadores, porque os profissionais só irão conquistar a autonomia do cuidador, quando este for incluído na equipe, levando em conta as questões legais que precisam ocorre no cuidado dominciliar. Em alguns casos, as atividades realizadas por cuidadores informais em casa são muito complicadas, e são realizadas sem a supervisão de profissionais com a capacidade legal, o que pode fazer com que ocorram danos ao atendimento do paciente e até mesmo situações de perigo para este (CARDOSO SMM, et al., 2020).

Ademais, para atender de maneira integral toda e qualquer demanda trazidas pela população idosa, se sugere, além dos serviços de atenção domiciliar ofertados pelo Sistema Único de Saúde, o desenvolvimento de um centro de prestação de atividades de apoio sociais no domicílio, os quais ofertam uma variedade de serviços para atender as necessidades da pessoa idosa, desde realizar limpeza e higiene, serviços de entregas até monitoramento a uma distância considerável, entre outros, gerando assim uma folga para os cuidadores principais sem deixar o idoso em situações de risco. Frente a esses pressupostos, e por averiguar a importância da visão de toda a equipe que trabalha no SUS que exercem o atendimento no domicílio do paciente, compreende-se os sentidos das ações da atenção domiciliar no âmbito da Atenção Básica à atenção a pessoa idosa (POZZOLI SML, et al., 2017; MARQUES FP, et al., 2018).

\section{CONSIDERAÇÕES FINAIS}

O bem-estar da população idosa é um fator primordial a ser considerado nas discussões de políticas públicas. Desse modo, sabe-se que o direito à saúde é garantido à população brasileira e independe da situação financeira de cada cidadão. Além disso, é dessa forma que ocorre o fortalecimento do SUS, sendo conjunta às operadoras privadas de plano de saúde, pois assim conseguem prestar integralidade na assistência. Além do que foi supracitado, as UBSs devem ser adaptadas para receber e atender os idosos, desde a infraestrutura até a equipe professional, uma vez que se percebe que no sistema de saúde. Destarte que, o presente estudo relatando que devido ao aumento de idosos no Brasil, se faz necessário uma atividade socioeducativa com as equipes multiprofissionais da UBS para que estas sejam capacitadas a atender esses pacientes e entender as suas vulnerabilidades.

\section{REFERÊNCIAS}

1. ALMEIDA APSC, et al. Falta de acesso e trajetória de utilização de serviços de saúde por idosos brasileiros. Ciência e Saúde Coletiva, 2020; 25(6).

2. BANDEIRA EMFS, et al. Atenção à saúde do idoso. Governo Federal, 2014. 46 p.

3. BARROS RLM, et al. Violência doméstica contra idosos assistidos na atenção básica. Saúde Debate, 2019; 43(122).

4. BENITO LAO, AQUINO EB. Modalidades assistenciais de atendimento ao idoso: revisão da literatura. Ciências da Saúde, 2016; 14(2).

5. CARDOSO SMM, et al. Cuidadores de idosos em estratégias de saúde da família: o estresse destes indivíduos. Revista Eletrônica Acervo saúde, 2020; Sup. 49: e3971.

6. COELHO LP, et al. Rede de atenção ao idoso: Fatores facilitadores e barreiras para implementação. Revista de Saúde Coletiva, 2019; 28(4).

7. SILVA RM, et al. Desafios e possibilidades dos profissionais de saúde no cuidado do idoso dependente. Revista de Ciência e Saúde Coletiva, 2021; 26(1).

8. ANDRADE FLJP, et al. Incidência e fatores de risco para hospitalização em pessoas idosas institucionalizadas. Revista Brasileira de Geriatria e Gerontologia, 2021; 23(4). 
9. CASTRO VC, et al. Violência contra os idosos brasileiros: Uma análise das internações hospitalares. Revista Brasileira de Enfermagem, 2017; 71(2).

10. MORAES EN, et al. Saúde da pessoa Idosa. SUS, 2019.

11. LUBNOW JAM, SILVA AO. O que os idosos pensam sobre o atendimento nos serviços de saúde. Revista Brasileira de Geriatria e Gerontologia, 2019; 22(2).

12. MARQUES AEF, et al. Assistência farmacêutica: uma reflexão sobre o papel do farmacêutico na saúde do paciente idoso no brasil. Temas em Saúde, 2017; 17(3).

13. MARQUES FP, BULGARELLI AF. Os sentidos da atenção domiciliar no cuidado ao idoso na finitude: A perspectiva humana do profissional do SUS. Revista de Ciência e Saúde Coletiva, 2020; 25(6).

14. OLIVEIRA KSM, et al. Violência contra idosos: concepções dos profissionais de enfermagem acerca da detecção e prevenção. Rev. Gaúcha Enferm. 2018; 39.

15. PLACIDELI N, NEMES MIB. Avaliação da atenção integral ao idoso em serviços de atenção primária. Revista de Saúde Pública, 2020; 54.

16. POZZOLI SML, CECÍLIO LCO. Sobre o cuidar e ser cuidado na atenção domiciliar. Saúde Debate. Rio de Janeiro, $2017 ; 41(115)$.

17. RAMOS FP, et al. Fatores associados à depressão em idosos. Revista Eletrônica Acervo saúde, 2019; Sup. 19 : e239.

18. RAMOS LV, et al. Caderneta de Saúde da pessoa Idosa na Atenção Primária: Uma revisão integrativa. Humanidades e Inovação, 2019; 6(2); 1-9.

19. RÊGO AS, et al. Fatores associados ao atendimento a idosos por condições sensíveis à Atenção Primária à Saúde. Revista Brasileira de Geriatria e Gerontologia, 2017; 20(6).

20. RODRIGUES MM, et al. tendência das internações e da mortalidade de idosos por condições sensíveis à atenção primária. Revista Brasileira de Epidemiologia, 2019; 22.

21. SCHENKE M, Da Costa DH. Os avanços e desafios da atenção à saúde da população idosa com doenças crônicas na atenção Primária à Saúde. Ciência e Saúde Coletiva, 2019; 24(4).

22. SILVA AMM, et al. Uso de serviços de saúde por idosos brasileiros com e sem limitação funcional. Revista de Saúde Pública, 2017; 51(1).

23. SOUSA EA, et al. Avaliação da atenção primária à saúde: qualidade da coordenação do serviço na perspectiva do idoso. Revista de Saúde Pública, 2019, 11(9).

24. TAVARES RE, et al. Envelhecimento saudável na perspectiva de idosos: uma revisão integrativa. Revista Brasileira de Geriatria e Gerontologia, 2017; 20(6).

25. THUM C, et al. Perfil de idosos e sua percepção enquanto satisfação nos serviços de assistência do sus na atenção básica. Revista Interdisciplinar de Estudos em Saúde, 2019; 8(2).

26. VIANNA LG, et al. Relação Médico-Paciente idoso: desafios e perspectivas. Revista Brasileira de Educação Médica, $2009 ; 34(1)$. 